Produto \& Produção, vol. 6, n. 1, p. 01-08, fev. 2002

\title{
Auxílio à decisão para a adoção de políticas de compras
}

\author{
Valério Antônio Pamplona Salomon \\ Doutorando em Engenharia pelo Departamento de Engenharia de Produção \\ Escola Politécnica da Universidade de São Paulo (Poli/USP) \\ Professor Assistente do Departamento de Produção \\ Faculdade de Engenharia de Guaratinguetá, Universidade Estadual Paulista (FEG/UNESP)
}

\begin{abstract}
O presente artigo relata os resultados de uma pesquisa sobre a aplicação do auxílio à decisão na adoção de políticas de compras. Foram desenvolvidos estudos sobre o processo de compras e sua inerente tomada de decisão, em situações já existentes, assim como novas proposições literárias e novas práticas profissionais. Uma política de compra, recentemente estabelecida por empresa multinacional do ramo automobilístico, foi investigada e melhorias para sua execução foram, finalmente, propostas.
\end{abstract}

Palavras-chave: auxílio à decisão; compras; gerência da qualidade.

This article reports results from a research involving decision support applied to procurement policies. There was developed some studies about the procurement process and its necessary decision making, under preexistent situations and also others newly proposed or professionally adopted. A new procurement policy recently developed by a multinational car manufacturing company was investigated in order to improve its performance.

Keywords: decision support; procurement; quality management.

\section{Introdução}

Um dos responsáveis pela introdução da Gerência pela Qualidade Total (TQM, Total Quality

Management) no Brasil, o Prof. Vicente Falconi Campos observou que "os métodos com que muitas empresas brasileiras atuam no setor de compras são, de fato, inadequados" (Campos, 1992). Por outro lado, os processos de compras têm sua importância aumentada pelas atuais tendências do mercado mundial: a terceirização aumenta o número de itens comprados por uma empresa; a globalização exige que as empresas se tornem "companhias de classe mundial, capazes de fornecer e comprar peças, equipamentos e serviços de todos e para todos os pontos do mundo" (Marinho \& Amato Neto, 1997).

Uma prática muito utilizada, a compra pelo menor preço, é condenada pelo "quarto ponto" de Deming (1986): o menor preço de um componente ou matéria-prima não implica, necessariamente, em menor "custo" de aquisição. Isto se dá por uma razão muito simples: um fornecedor que oferece o menor preço nem sempre pode ser considerado o mais confiável (no prazo de entrega ou na qualidade do produto, por exemplo). À tentativa de se evitar a compra pelo menor preço, denomina-se política de compra. Existe na literatura uma boa variedade de políticas de compras. Destacam-se a "formação de preços baseada em incentivos à qualidade" (Windham, 1995), a "planilhas de fornecedores" (Desai, 1996) e a "seleção de fornecedores pelo custo total mínimo" (Quelhas \& Qassim, 1996). Das peculiaridades de cada política apresentada na Tabela 1 , surgem vantagens e desvantagens que devem ser ponderadas na escolha por uma ou mais política de compra. Entretanto, todas estas políticas possuem uma inconveniência em comum: a adoção, de maneira arbitrária, de importantes parâmetros. Esta arbitrariedade poderá se transformar numa barreira para empresas que optem pela utilização da política de compra, mas não possuam conhecimento para especificar os valores destes parâmetros.

Utilizando ferramentas de auxílio à decisão, Salomon (1998) eliminou a arbitrariedade na adoção dos parâmetros da política "formação de preços baseada em incentivos à qualidade" em um caso envolvendo indústria do ramo aeronáutico. O objetivo do presente trabalho é avaliar a aplicação do auxílio à decisão na adoção de outra política de compra, em situação distinta e comparar os resultados. Na próxima seção será apresentado um método de auxílio à decisão que se acredita ser útil para tal. 
Tabela 1 - Políticas de compras

\begin{tabular}{|l|l|l|}
\hline \multicolumn{1}{|c|}{ Política } & \multicolumn{1}{|c|}{ Pontos fortes } & \multicolumn{1}{c|}{ Pontos fracos } \\
\hline $\begin{array}{l}\text { Formação de preços } \\
\text { baseada em incentivos } \\
\text { à qualidade }\end{array}$ & $\begin{array}{l}\text { Alteração no preço final a ser } \\
\text { pago ao fornecedor com base na } \\
\text { qualidade do produto comprado } \\
\text { (originalmente, propôs-se } \\
\text { estimar o nível de qualidade do } \\
\text { produto pela média dos índices } \\
\text { de capabilidade de seus vários } \\
\text { processos) }\end{array}$ & $\begin{array}{l}\text { O produto deve possuir } \\
\text { características mensuráveis ou, no } \\
\text { míno, facilmente quantificáveis e } \\
\text { para estas deverão existir (ou se } \\
\text { estabelecer) valores nominais e de } \\
\text { tolerância }\end{array}$ \\
\hline $\begin{array}{l}\text { Planilhas de } \\
\text { fornecedores }\end{array}$ & $\begin{array}{l}\text { Cadastro e acompanhamento do } \\
\text { desempenho de fornecedores } \\
\text { quanto a critérios quantitativos } \\
\text { (preço, prazo de entrega, itens } \\
\text { defeituosos, etc.) e qualitativos } \\
\text { (suporte técnico, tecnologia do } \\
\text { produto, etc.) }\end{array}$ & $\begin{array}{l}\text { Provável alta variabilidade dos pesos } \\
\text { dos critérios quando houver vários } \\
\text { produtos em uma única fábrica }\end{array}$ \\
\hline $\begin{array}{l}\text { Seleção de } \\
\text { fornecedores pelo } \\
\text { custo total mínimo }\end{array}$ & $\begin{array}{l}\text { Alteração no preço final com } \\
\text { base na quantificação de itens } \\
\text { como atendimento, garantia, } \\
\text { prazo de entrega, características } \\
\text { opcionais, etc. }\end{array}$ & $\begin{array}{l}\text { O fato da alteração no preço poder } \\
\text { ser "para cima" ou "para baixo" do } \\
\text { preço real causa impactos como } \\
\text { lucro, ou prejuízo, para ambos } \\
\text { fornecedor e comprador }\end{array}$ \\
\hline
\end{tabular}

\section{Auxílio à decisão por múltiplos critérios}

Um método de auxílio à decisão por múltiplos critérios (MCDM, Multiple-Criteria Decision Method), como o próprio nome sugere, é utilizado em situações em que se considera mais de um critério, por exemplo: custo, qualidade, atendimento, etc. Basicamente, estes métodos trabalham com a mesma ferramenta principal, a matriz de decisão. A Tabela 2 representa a matriz de decisão utilizada em uma situação em que se deseja analisar três alternativas de acordo com cinco critérios diferentes.

Tabela 2 - Matriz de decisão

\begin{tabular}{|l|c|c|c|c|c|}
\cline { 2 - 6 } \multicolumn{1}{c|}{} & Critério 1 & Critério 2 & Critério 3 & Critério 4 & Critério 5 \\
\hline Alternativa 1 & $\mathrm{a}_{11}$ & $\mathrm{a}_{12}$ & $\mathrm{a}_{13}$ & $\mathrm{a}_{14}$ & $\mathrm{a}_{15}$ \\
\hline Alternativa 2 & $\mathrm{a}_{21}$ & $\mathrm{a}_{22}$ & $\mathrm{a}_{23}$ & $\mathrm{a}_{24}$ & $\mathrm{a}_{25}$ \\
\hline Alternativa 3 & $\mathrm{a}_{31}$ & $\mathrm{a}_{32}$ & $\mathrm{a}_{33}$ & $\mathrm{a}_{34}$ & $\mathrm{a}_{35}$ \\
\hline
\end{tabular}

Na matriz de decisão, os $a_{i j}$ representam o desempenho das alternativas $i$ segundo os critérios $j$. A maneira com que um MCDM trabalha os $a_{i j}$ é que o torna diferente dos demais. Métodos como o ELECTRE (Elimination and Choice Translating Reality) fornecem apenas a ordenação (ranking) das alternativas com base em princípios de dominância. Outros métodos fornecem, além desta ordenação, uma medida do desempenho das alternativas, considerando todos os critérios (desempenho global). Dentre os vários métodos que fornecem valores de desempenho global das alternativas, já utilizados com sucesso em diversas situações, podem ser citados:

- AHP (Analytic Hierarchy Process), proposto pelo Prof. Thomas L. Saaty (1977);

- ANP (Analytic Network Process), também desenvolvido por Saaty (1996);

- FDA (Fuzzy Decision Approach), baseada em conjuntos Fuzzy e proposta por Liang \& Wang (1992);

- MACBETH (Measuring Attractiveness by a Categorical Based Evaluation Technique), proposto por Bana e Costa \& Vasnick (1994);

- TOPSIS (Technique for Order Preference by Similarity to Ideal Solution), cujo desenvolvimento se deve a Hwang \& Yoon (1981).

Da experiência observada em aplicações de MCDM, Salomon \& Montevechi (2001) recomendam a utilização do AHP, esperando-se a obtenção de bons resultados, desde que se satisfaçam três pré-requisitos: disponha-se de tempo para a tomada de decisão; não existam mais de nove alternativas; haja independência entre os elementos de um mesmo nível hierárquico.

\subsection{Origem do método AHP}

O AHP se originou a partir do trabalho do Prof. Thomas L. Saaty, na década de 70: a solução de um conflito militar no Oriente Médio (Palmer, 1999). 
Naquela época, o Prof. Saaty atuou como consultor do governo egípcio e na análise da situação utilizouse da Teoria dos Jogos. Embora o governo egípcio tenha seguido sua orientação, o que acabou ocasionando um processo de paz, o Prof. Saaty não ficou satisfeito com a maneira que se chegou à decisão. Aspectos importantes não foram considerados por serem intangíveis, ou seja, não quantificáveis.

Para desenvolver o AHP, o Prof. Saaty baseou-se em conceitos de Álgebra Linear, Pesquisa Operacional e Psicologia. Os resultados foram publicados em periódico de Matemática e Psicologia (Saaty, 1977). Logo em seguida, o método começou a ser investigado por pesquisadores de diversas áreas, incluindo Engenharia e Administração. Na mesma época, foi lançado um modelo computacional (o software Expert Choice), desenvolvido pelo Prof. Ernest Forman consultor e parceiro do Prof. Saaty na empresa Incorporated Expert Choice. Atualmente, a empresa possui clientes como IBM, Xerox, NASA, General Motors, 3M, etc. utilizando seu software que já está na versão 9.0. Lombardo (1996) identificou mais de 1.500 trabalhos acadêmicos que descrevem o AHP, trazem aplicações, críticas, aperfeiçoamentos, etc.

\subsection{Fases de execução do AHP}

O AHP é executado através de três fases: estruturação do modelo, realização de julgamentos e síntese dos resultados. A estruturação, consiste na obtenção do modelo de decisão, que no AHP possui a forma de uma hierarquia. A Figura 1 traz o exemplo de um modelo, com três níveis hierárquicos.

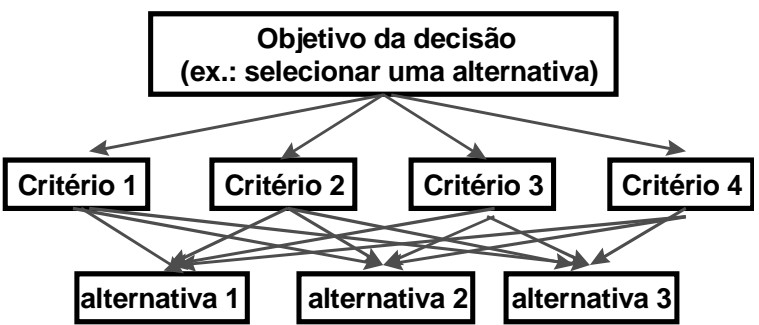

Figura 1 - Estrutura de decisão hierárquica em três níveis

Na fase seguinte do AHP, o desempenho global de cada alternativa é obtido ponderando-se o desempenho da alternativa em cada critério pelo peso do critério. O peso do critério e o desempenho das alternativas em cada critério são estimados pelos componentes do vetor de prioridades obtidos através de estimativa do autovetor $\mathbf{V}$ da equação 1, onde $\mathbf{A}$ é a matriz de julgamentos e $\lambda_{\mathrm{MAX}}$ é o máximo autovalor.

$$
\mathbf{A} \times \mathbf{V}=\lambda_{\text {MAX }} \times \mathbf{V}
$$

A Tabela 3 apresenta um problema clássico de estimação de distância, proposto por Saaty (1991). Neste problema desejava-se saber qual cidade estava mais distante da Filadélfia e utilizou-se para isto a opinião de um passageiro aéreo experiente que se baseou no seu cansaço após o vôo, utilizando-se da escala fundamental (proposta por Saaty, 1977) de 1 a 9. Neste caso, julgou-se que Filadélfia está "levemente" mais distante de Tóquio do que Cairo e "absolutamente" mais distante de Tóquio do que de Chicago.

Tabela 3 - Matriz de julgamentos para estimação da distância à Filadélfia

\begin{tabular}{|lc|c|c|c|c|c|}
\cline { 2 - 7 } \multicolumn{1}{c|}{} & CAI & TYO & ORD & SFO & LGW & YMX \\
\hline Cairo (CAI) & 1 & $1 / 3$ & 8 & 3 & 3 & 7 \\
\hline Tóquio (TYO) & 3 & 1 & 9 & 3 & 3 & 9 \\
\hline Chicago (ORD) & $1 / 8$ & $1 / 9$ & 1 & $1 / 6$ & $1 / 5$ & 2 \\
\hline $\begin{array}{l}\text { San Francisco } \\
\text { (SFO) }\end{array}$ & $1 / 3$ & $1 / 3$ & 6 & 1 & $1 / 3$ & 6 \\
\hline Londres (LGW) & $1 / 3$ & $1 / 3$ & 5 & 3 & 1 & 6 \\
\hline Montreal (YMX) & $1 / 7$ & $1 / 9$ & $1 / 2$ & $1 / 6$ & $1 / 6$ & 1 \\
\hline
\end{tabular}

Hoje em dia, existem vários modelos comerciais de software (MATLAB, MATCAD, etc.) capazes de calcular o autovetor da matriz de uma dada equação. Porém, como a matriz A é uma matriz recíproca positiva, há como se estimar o autovetor da equação 1 , através de cálculos simples. Uma delas é estimando-se cada componente de $\mathbf{P}$ a partir da média geométrica dos elementos da correspondente linha de A. Para os valores da Tabela 3, temos:

$$
\mathbf{P}=\left[\begin{array}{l}
2,35 \\
3,60 \\
0,31 \\
1,05 \\
1,47 \\
0,25
\end{array}\right]
$$

O autovetor pode ser normalizado para que a soma de seus elementos seja igual a 1 . Assim, P' pode ser obtido através da equação 2 , para $i=1,2,3, \ldots n$.

$$
\boldsymbol{p}_{i}=\frac{p_{i}}{\sum_{i=1}^{n} p_{i}}
$$

Logo:

$$
\mathbf{P}^{\prime}=\left[\begin{array}{l}
0,260 \\
0,399 \\
0,035 \\
0,116 \\
0,163 \\
0,027
\end{array}\right]
$$


A Tabela 4 traz uma comparação dos dados reais (distância a Filadélfia em milhas) com os elementos de P'. Observa-se que, realmente, houve uma proximidade entre os resultados obtidos com a utilização do $\mathrm{AHP}$ e os valores reais.

Tabela 4 - Comparação dos resultados obtidos com a distância à Filadélfia

\begin{tabular}{|l|c|c|c|}
\cline { 2 - 4 } \multicolumn{1}{c|}{} & $\begin{array}{c}\text { Distância à } \\
\text { Filadélfia } \\
\text { (milhas) }\end{array}$ & $\begin{array}{c}\text { Distância } \\
\text { normalizada }\end{array}$ & $\mathrm{P}^{\prime}$ \\
\hline Cairo & 5.729 & 0,278 & 0,260 \\
\hline Tóquio & 7.449 & 0,361 & 0,399 \\
\hline Chicago & 660 & 0,032 & 0,035 \\
\hline $\begin{array}{l}\text { San } \\
\text { Francisco }\end{array}$ & 2.732 & 0,132 & 0,116 \\
\hline Londres & 3.658 & 0,177 & 0,163 \\
\hline Montreal & 400 & 0,019 & 0,027 \\
\hline
\end{tabular}

Uma grande vantagem do AHP sobre outros MCDMs é a verificação da coerência dos julgamentos. Uma matriz de julgamentos coerentes deverá satisfazer a equação 3 , para todos $i, j=1,2,3, \ldots n$ :

$$
a_{i j}=\frac{p_{i}}{p_{j}}
$$

Se a equação 6 for satisfeita deveremos ter $\lambda_{\text {MAX }}$ igual a $n$. Quando um valor de $a_{i j}$ não satisfizer (3), $\lambda_{\text {MAX }}$ será maior que $n$ (Saaty, 1977). Para os julgamentos apresentados na Tabela 3, temos:

$$
\lambda_{\text {MAX }}=6,49
$$

Uma medida da coerência dos julgamentos é o índice de coerência, $c_{I}$, que mede o afastamento entre $\lambda_{\text {MAX }} \mathrm{e}$ $n$ :

$$
c_{I}=\frac{\lambda_{M A X}-n}{n-1}
$$

Uma medida melhor (Saaty, 1991) é a razão de coerência, $c_{R}$, que considera também um "erro aleatório" associado à ordem da matriz de julgamentos:

onde $c_{A}$ é a coerência aleatória obtida pela tabela 5 .

Tabela 5 - Coerência aleatória

\begin{tabular}{|c|c|c|c|c|c|c|c|c|}
\hline $\mathrm{n}$ & 2 & 3 & 4 & 5 & 6 & 7 & 8 & 9 \\
\hline $\mathrm{c}_{\mathrm{A}}$ & 0 & 0,52 & 0,89 & 1,11 & 1,25 & 1,35 & 1,4 & 1,45 \\
\hline
\end{tabular}

Substituindo valores nas equações 4 e 5, obtêm-se:

$$
\begin{gathered}
c_{I}=\frac{6,49-6}{6-1}=0,098 \\
c_{R}=\frac{0,098}{1,25}=0,078
\end{gathered}
$$

Para valores de $c_{R}$ menores ou iguais a 0,10 , o autovetor P poderá ser aceito (Saaty, 1991). Acima deste valor os julgamentos do especialista são considerados incoerentes e merecem ser revistos.

\section{A política de compra IQTC}

O caso a ser apresentado refere-se a uma unidade industrial de uma empresa especializada no ramo automobilístico (produção de caminhões, veículos utilitários e de passeio) localizada na cidade paulista de São Bernardo de Campo. O escopo do estudo se deu na Diretoria de Compras de Material, precisamente, na Gerência de Compras de Material Produtivo. As instruções de trabalho, organizadas em fluxogramas, constam do Manual de Compras, documento interno da empresa.

Seguindo suas instruções de trabalho, o comprador deve selecionar o fornecedor para um determinado pedido de acordo com um Índice Qualitativo Técnico

\begin{tabular}{|c|c|c|c|}
\hline & $\begin{array}{c}\text { Critérios } \\
\text { específicos }\end{array}$ & Aspecto avaliado & $\begin{array}{c}\text { Pontuação } \\
\text { máxima }\end{array}$ \\
\hline \multirow{4}{*}{$\begin{array}{l}\text { Conceito } \\
\text { técnico }\end{array}$} & IQD & Dimensional & 10 \\
\hline & IQMP & $\begin{array}{l}\text { Materiais e } \\
\text { processos }\end{array}$ & 10 \\
\hline & IQRC & $\begin{array}{l}\text { Reclamações de } \\
\text { campo e garantia }\end{array}$ & 10 \\
\hline & ISQ & $\begin{array}{c}\text { Certificação do } \\
\text { sistema de qualidade }\end{array}$ & 10 \\
\hline \multirow{4}{*}{$\begin{array}{l}\text { Conceito } \\
\text { comercial }\end{array}$} & IQDP & $\begin{array}{l}\text { Desenvolvimento de } \\
\text { produtos }\end{array}$ & 10 \\
\hline & IQC & Compras & 20 \\
\hline & IQL & Logística & 20 \\
\hline & IQF & Financeiro & 10 \\
\hline \multicolumn{3}{|c|}{ IQTC } & 100 \\
\hline
\end{tabular}
e Comercial (IQTC) deste fornecedor. O IQTC é uma nota atribuída aos fornecedores com base em outros índices, conforme Tabela 6 e Tabela 7.

Tabela 6 - Cálculo do IQTC 
Tabela 7 - Índices que compõe o IQTC

\begin{tabular}{|c|c|c|}
\hline & Significado & Bases de cálculo \\
\hline IQD & $\begin{array}{l}\text { Índice de qualidade } \\
\text { dimensional }\end{array}$ & $\begin{array}{l}\text { Número médio de peças defeituosas por } \\
\text { produto }\end{array}$ \\
\hline IQMP & $\begin{array}{l}\text { Índice de qualidade de } \\
\text { materiais e processos }\end{array}$ & Capacidade tecnológica \\
\hline IQRC & $\begin{array}{l}\text { Índice de qualidade em } \\
\text { garantia }\end{array}$ & $\begin{array}{l}\text { Número de reclamações por período, recall e } \\
\text { grau de parceria }\end{array}$ \\
\hline ISQ & $\begin{array}{l}\text { "Índice" de sistema d4 } \\
\text { qualidade }\end{array}$ & $\begin{array}{l}\text { Mérito para empresas com sistema da } \\
\text { qualidade reconhecido por órgãos } \\
\text { certificadores }\end{array}$ \\
\hline IQDP & $\begin{array}{l}\text { Índice de qualidade em } \\
\text { desenvolvimento de } \\
\text { produtos }\end{array}$ & $\begin{array}{l}\text { Pronto atendimento a consultas, qualidade da } \\
\text { documentação de engenharia e disponibilidade } \\
\text { de tecnologia para aplicação em novos } \\
\text { produtos }\end{array}$ \\
\hline IQC & $\begin{array}{l}\text { Índice de qualidade em } \\
\text { compras }\end{array}$ & $\begin{array}{l}\text { Qualidade na apresentação, negociação e } \\
\text { confiabilidade da oferta }\end{array}$ \\
\hline IQL & $\begin{array}{l}\text { Índice de qualidade em } \\
\text { logística }\end{array}$ & $\begin{array}{l}\text { Méritos e deméritos operacionais como } \\
\text { pontualidade no fornecimento }\end{array}$ \\
\hline IQF & $\begin{array}{l}\text { Índice de qualidade } \\
\text { financeira }\end{array}$ & $\begin{array}{l}\text { Receita operacional líquida, do patrimônio } \\
\text { líquido e de outros indicadores econômicos }\end{array}$ \\
\hline
\end{tabular}

Através de avaliações trimestrais os fornecedores são classificados de acordo com um conceito apresentado na Tabela 8. Um fornecedor "ótimo" terá preferência num pedido contra um fornecedor "bom" ou "regular". Fornecedores "ruins" e "péssimos" não se encontram em condições de fornecimento. Mas, o mais importante é que os avaliadores de cada índice deverão entrar em contato com os fornecedores, quando se identificar pontos que requerem melhorias, estabelecendo-se trabalhos conjuntos ou plano de ações.

Tabela 8 - Classificação de fornecedores

\begin{tabular}{|r|l|}
\hline Resultado & Conceito \\
\hline IQTC $\geq 80$ & Ótimo \\
\hline $70 \leq$ IQTC $<80$ & Bom \\
\hline $50 \leq$ IQTC $<70$ & Regular \\
\hline $40 \leq$ IQTC $<50$ & Ruim \\
\hline IQTC $<40$ & Péssimo \\
\hline
\end{tabular}

Apesar da boa política de compra adotada pela empresa, seus compradores, freqüentemente, se vêem realizando retrabalhos: alguns fornecedores não conseguiam atender determinados pedidos para os quais eram escolhidos. Havia casos de empresas que caminhavam para a falência, mas ainda apresentavam um bom IQTC, superior ao de outras financeiramente mais sólidas. No caso extremo de falência de um fornecedor, o pedido de fornecimento deverá ser refeito para outro fornecedor a fim de evitar o atraso ou o não atendimento de um pedido posterior da cadeia de fornecimento. Este fato leva ao questionamento dos pesos dos critérios que compõem o IQTC (Tabela 6).

\section{Aplicação do auxílio à decisão}

De acordo com o apresentado, nota-se que o IQTC já possui a estrutura hierárquica apresentada na Figura 2. Propõe-se, então, a utilização do método AHP como uma maneira melhor para atribuição dos pesos dos critérios que compõem o IQTC, com base na experiência de casos ocorridos na empresa.

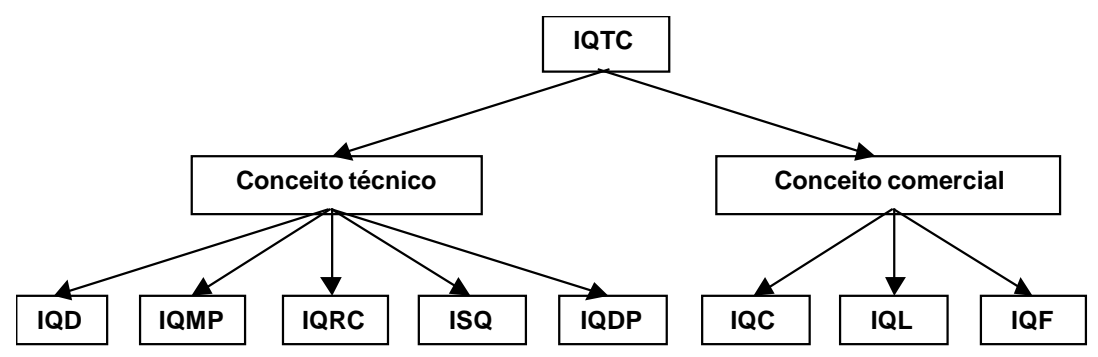

Figura 2 - Estrutura hierárquica do IQTC 
A premissa que levou aos pesos usualmente praticados para cada índice foi que os conceitos técnico e comercial deveriam possuir o mesmo peso, ou seja, $50 \%$. Isto ocasionou o fato dos índices IQC e IQL possuírem pesos duas vezes maiores que os demais. Entretanto, através de entrevistas com os responsáveis das áreas envolvidas com o gerenciamento do IQTC, notou-se que o conceito técnico deveria possuir, na verdade, uma "importância moderadamente maior" que o conceito comercial, justificando o valor 2 (conforme a "escala fundamental" de Saaty, 1977) na célula 2-1 da matriz de julgamentos apresentada na Tabela 9. A prioridade relativa de cada conceito foi obtida através de estimação do autovetor da matriz de julgamentos.

Tabela 9 - Julgamentos para a importância dos conceitos

\begin{tabular}{|l|c|c|c|}
\cline { 2 - 4 } \multicolumn{1}{c|}{} & $\mathrm{C}$ & $\mathrm{T}$ & Prioridade relativa \\
\hline Conceito comercial $(\mathrm{C})$ & 1 & $1 / 2$ & 0,333 \\
\hline Conceito técnico $(\mathrm{T})$ & 2 & 1 & 0,667 \\
\hline
\end{tabular}

As importâncias relativas dos índices associados aos conceitos comercial e técnico receberam os julgamentos apresentados na Tabela 10 e na Tabela 11, respectivamente.

Tabela 10 - Julgamentos para a importância relativa dos índices associados ao conceito comercial

\begin{tabular}{|c|c|c|c|c|}
\cline { 2 - 5 } \multicolumn{1}{c|}{} & IQC & IQL & IQF & Prioridade relativa \\
\hline IQC & 1 & 2 & $1 / 2$ & 0,311 \\
\hline IQL & $1 / 2$ & 1 & $1 / 2$ & 0,196 \\
\hline IQF & 2 & 2 & 1 & 0,493 \\
\hline
\end{tabular}

Tabela 11 - Julgamentos para a importância relativa dos índices associados ao conceito técnico

\begin{tabular}{|c|c|c|c|c|c|c|}
\cline { 2 - 7 } \multicolumn{1}{c|}{} & IQD & IQMP & IQRC & ISQ & IQDP & Prioridade relativa \\
\hline IQD & 1 & 1 & $1 / 5$ & 1 & $1 / 3$ & 0,090 \\
\hline IQMP & 1 & 1 & $1 / 5$ & 1 & $1 / 3$ & 0,090 \\
\hline IQRC & 5 & 5 & 1 & 5 & 2 & 0,468 \\
\hline ISQ & 1 & 1 & $1 / 5$ & 1 & $1 / 3$ & 0,090 \\
\hline IQDP & 3 & 3 & $1 / 2$ & 3 & 1 & 0,261 \\
\hline
\end{tabular}

Ressalta-se que os julgamentos apresentados na Tabela 10 e na Tabela 11 possuem alto grau de coerência, o que pode ser constatado com valores de $c_{R}$, respectivamente, iguais a 0,0448 e 0,0012 . Este alto grau de coerência pode ser explicado pelo fato dos julgamentos terem sido realmente realizados por especialistas.

A Tabela 12 consolida a proposta para novo cálculo do IQTC, lembrando-se que o IQTC máximo deverá ser de 100 pontos para poder-se continuar trabalhando com a classificação apresentada na Tabela 8, além de toda a filosofia de melhoria contínua que seu emprego propicia. Observa-se que o índice financeiro (IQF) passaria a contribuir com 16,4 pontos (contra os 10 da tabela 6), em contrapartida o índice dimensional (IQD), o índice de materiais e processos (IQMP) e o índice de certificação da qualidade (ISQ) reduziriam sua contribuição de 10 para 6,0 pontos.

Tabela 12 - Proposta para cálculo do IQTC

\begin{tabular}{|c|c|c|}
\cline { 2 - 3 } \multicolumn{1}{c|}{} & Prioridade global & Pontuação máxima \\
\hline IQD & $0,667 \times 0,090=0,060$ & $0,060 \times 100=6,0$ \\
\hline IQMP & $0,667 \times 0,090=0,060$ & $0,060 \times 100=6,0$ \\
\hline IQRC & $0,667 \times 0,468=0,313$ & $0,313 \times 100=31,3$ \\
\hline ISQ & $0,667 \times 0,091=0,061$ & $0,061 \times 100=6,1$ \\
\hline IQDP & $0,667 \times 0,261=0,174$ & $0,174 \times 100=17,4$ \\
\hline IQC & $0,333 \times 0,311=0,104$ & $0,104 \times 100=10,4$ \\
\hline IQL & $0,333 \times 0,196=0,065$ & $0,065 \times 100=6,5$ \\
\hline IQF & $0,333 \times 0,493=0,164$ & $0,164 \times 100=16,4$ \\
\hline Total & 1 & 100 \\
\hline
\end{tabular}

Um fornecedor real possuía desempenho nos diversos índices conforme apresentado na tabela 13 . O impacto das modificações propostas pode ser visualizado na última linha desta tabela: na pontuação atual do IQTC (Tabela 6) tal fornecedor recebe nota 76 (sendo considerado um "bom fornecedor"); com base na pontuação proposta (Tabela 12) sua nota cairia para 66,7 e seu conceito também cairia de "bom fornecedor" para "fornecedor regular".

Tabela 13 - Dados reais do desempenho de um fornecedor

\begin{tabular}{|c|c|c|c|}
\hline \multicolumn{2}{|c|}{ Fornecedor XX } & Cálculo atual & Cálculo proposto \\
\hline IQD & 8 & 8 & 4,80 \\
\hline IQMP & 7 & 7 & 4,20 \\
\hline IQRC & 6 & 6 & 18,78 \\
\hline ISQ & 10 & 10 & 6,10 \\
\hline IQDP & 6 & 6 & 10,44 \\
\hline IQC & 9 & 18 & 9,36 \\
\hline IQL & 8 & 16 & 5,20 \\
\hline IQF & 5 & 5 & 8,20 \\
\hline \multicolumn{2}{|c|}{ IQTC } & 76 & 67,08 \\
\hline
\end{tabular}

Pelo exemplo mostrado pode-se perceber que a proposta traduz uma melhoria da qualidade no procedimento de classificação de fornecedores: haverá uma escolha mais segura do ponto de vista financeiro. 


\section{Conclusões}

A aplicação do método AHP resultou em alteração na classificação de fornecedores. Mas, a grande vantagem desta aplicação foi o questionamento do processo de decisão em si, ou seja, a política de compras IQTC. Há de se louvar a gerência da empresa estudada pelas belas iniciativas de padronização do procedimento de compras e busca da melhoria contínua da produção desde a fonte. Entretanto, com a aplicação do método AHP, algumas premissas vieram a baixo. A primeira delas é que "conceito comercial" e "conceito técnico" deveriam receber o mesmo peso. Este fato não ocorre, pois como o conceito comercial possui um número menor de índices, alguns de seus índices recebem importância dobrada. Assim, numa seleção de fornecedores, o preço e o prazo de entrega (influentes nos índices IQC e IQL) podem acabar decidindo a favor de um fornecedor com sistema de qualidade não implantado, alto índice de refugos e péssima situação financeira. Estes últimos aspectos citados têm contribuído para a ocorrência de falhas na entrega prometida por diversos fornecedores.

Considera-se que o objetivo geral de "avaliar a aplicação do auxílio à decisão na adoção de novas políticas de compras" tenha sido atingido com a aplicação do método AHP em um procedimento de compra recentemente desenvolvido por empresa industrial do ramo automobilístico. A política de compras, implicitamente, abordada foi a "planilha de fornecedores". O método AHP se mostrou eficaz e eficiente, pois não foi necessária a utilização de outro método ou ferramenta de auxílio à decisão. Ao contrário do que aconteceu na eliminação da arbitrariedade na adoção dos parâmetros da política "formação de preços baseada em incentivos à qualidade", quando além do AHP também foram necessárias técnicas de conjuntos Fuzzy (Salomon, 1998).

Como tema para novas pesquisas surge a investigação mais detalhada da aplicação do método AHP: incluindo o desenvolvimento de rotinas para revisão de julgamentos, se for o caso, ou para redução do esforço necessário para se chegar à decisão (número de julgamentos). Não obstante, a comparação com aplicação de outros métodos quanto à eficiência e à eficácia são assuntos que também merecem ser abordados.

\section{Referências bibliográficas}

BANA E COSTA, C.; VANSNICK, J. C. MACBETH - an interactive path towards the construction of cardinal value functions, International Transactions in Operational Research, vol. 1, n. 4, 1994, p. 489500.
CAMPOS, V. F. TQC: Controle de Qualidade Total (no estilo japonês), Rio de Janeiro: Bloch Editores S. A., 1992.

DEMING, W. E. Out of Crisis, Cambridge (USA): Massachusetts Institute of Technology, 1986.

DESAI, M. P. Implementing a supplier scoreboard program, Quality Progress, vol. 29, n. 2, 1996, p. 7375 .

LIANG, G. e M. WANG A fuzzy multi-criteria decision method for facility selection, International Journal of Production Research, vol. 29, n. 11, 1991, p. 2313-1330.

LOMBARDO, S. B. References and Dissertations on the Analytic Hierarchy Process (1976-1996), Pittsburgh: University of Pittsburgh Press, 1996.

MARINHO, B. L.; AMATO NETO, J. A necessidade de gerenciamento da qualidade de fornecedores no ambiente globalizado, In: ENCONTRO NACIONAL DE ENGENHARIA DE PRODUÇÃO, 17., Anais em $C D$-ROM, Gramado: UFRGS \& Associação Brasileira de Engenharia de Produção, 1997.

PALMER, B. Click here for decisions, Fortune, vol. 139, n. 9, p. 153, 1999.

QUELHAS, O. L. G.; QASSIM, R. Y. Seleção de fornecedores baseado no critério do custo total mínimo, In: ENCONTRO NACIONAL DE ENGENHARIA DE PRODUÇÃO, 16., Anais em CD-ROM, Piracicaba: UNIMEP \& Associação Brasileira de Engenharia de Produção, 1996.

SAATY, T. L. A scaling method for priorities in hierarchical structures, Journal of Mathematical Psychology, vol. 15, 1997, p. 234-281.

SAATY, T. L. Método de Análise Hierárquica, tradução e revisão técnica: Wainer da Silveira e Silva, Rio de Janeiro: Makron Books do Brasil Editora Ltda. e Editora McGraw-Hill do Brasil, 1991.

SAATY, T. L. Decision Making with Dependence and Feedback: The Analytic Network Process, Pittsburgh: RWS Publications, 1996.

SALOMON, V. A. P. Contribuição para Auxílio à Decisão em Processos de Compras, dissertação de mestrado, Itajubá: Escola Federal de Engenharia de Itajubá, 1998. 
8

Produto \& Produção, vol. 6, n. 1 p. 01-08, fev. 2002

SALOMON, V. A. P. e MONTEVECHI, J. A. B. A

compilation of comparisons on the Analytic Hierarchy

Process and others multiple criteria decision making

methods: some cases developed in Brazil, In:

INTERNATIONAL SYMPOSIUM ON THE

ANALYTIC HIERARCHY PROCESS, Proceeding, p. 413-420, Berna (Suíça): Bern Universität, 2001.

WINDHAM, J. Implementing of Deming's fourth point, Quality Progress, vol. 28, n. 12, 1995, p. 43-

48.

ZADEH, L. A. Fuzzy sets, Information and Control, vol. 8,1965 , p. 177-200. 